\title{
Technical aspects of abdominal ultrasound and color Doppler assessment of bowel viability in necrotizing enterocolitis
}

\author{
Ricardo Faingold ${ }^{1}$ (D)
}

Received: 7 November 2017 /Revised: 14 December 2017 / Accepted: 9 January 2018 / Published online: 24 January 2018

(C) Springer-Verlag GmbH Germany, part of Springer Nature 2018

In this issue of Pediatric Radiology, Cuna et al. [1] presented their findings of a systematic review and meta-analysis on the performance of ultrasound (US) for the evaluation of necrotizing enterocolitis (NEC). The authors distinguished US findings that were or were not associated with a need for surgery or with death. The authors appropriately noted that additional study is needed to determine the optimal timing and frequency of US for NEC and to determine whether utilization of US improves outcomes of infants with NEC.

In light of this paper and noting an increasing interest in using US to evaluate for NEC, I would like to share my experience with abdominal US in neonates with NEC, particularly in regard to the technical aspects of bowel perfusion and viability assessment.

It is well established that NEC is one of the most common acute neonatal gastrointestinal conditions in the neonatal intensive care unit (NICU), affecting the preterm infant in about $90 \%$ of the cases. The prevalence is approximately $1-5 \%$ of admissions in the NICU [2-4]. NEC manifests in the first 2 weeks after birth or later [2]. The diagnosis of NEC might be delayed at times because the early signs are nonspecific [2-5]. The prognosis of NEC worsens with perforation, and mortality rates range from $15 \%$ to $30 \%$ [2-4].

Abdominal radiography is the standard modality for diagnosis, monitoring and guiding management in NEC. The use of abdominal US in NEC has been described in publications since the 1980s and 1990s [6-9]. It is performed at bedside and provides real-time imaging of the bowel, peritoneal fluid and other abdominal organs, without ionizing radiation.

The routine use of US in NEC has been increasing steadily all over the world in the last 12 years. In 2005, the use of color

Ricardo Faingold

ricardo.faingold@mcgill.ca

1 Department of Medical Imaging, Montreal Children's Hospital, McGill University Health Center,

1001 Boulevard Decarie, Montreal, QC H4A3J, Canada
Doppler was described to assess bowel viability in NEC [3]. This article described, for the first time, US and color Doppler US findings (bowel wall, peristalsis, mural perfusion) in the normal neonate and in NEC. The data were compared to abdominal radiography and modified Bell staging and correlated to outcomes. In this study, color Doppler US was more accurate than abdominal radiography in the assessment of transmural necrosis and contributed to the management of neonates with NEC, particularly the ones with moderate or moderate to severe disease (Bell stages 2B and 3A). Other studies also recently demonstrated the applications of US in NEC [10-13].

A comprehensive description of technique and protocol should be made in order to standardize the exams. Attention to technique and standard protocol is crucial to obtain reproducible and reliable examinations. All neonates must be monitored by a bedside nurse throughout the exam. The exam should be stopped in case of desaturation or any signs of instability. Placing the transducer over the abdomen should be done gently because the child might become clinically unstable in case of tenderness and peritonitis. I recommend working closely with the nurses to mobilize and position the babies safely. The exam takes approximately 15-20 min.

At my institution, the US examination is divided into two parts using a linear-array transducer $(12-20 \mathrm{MHz})$. Initially, a brief abdominal sonography is performed to assess the relationship of the superior mesenteric artery and superior mesenteric vein, portal venous gas, free fluid and its character, and free air. Peritoneal fluid is considered complex when echogenic material or septations are noted.

Subsequently, the bowel loops are evaluated. The abdomen should be divided topographically into four quadrants. All quadrants are interrogated in gray-scale and color Doppler US in real time.

Gray-scale evaluation consists of assessment of peristalsis, bowel wall echotexture, wall thickness and presence of pneumatosis intestinalis. Color Doppler US is used to evaluate intestinal mural blood flow with a standard protocol and 
parameters using the lowest possible pulse repetition frequency without aliasing, and color gain of 40 or the highest color Doppler gain settings without flash artifacts. Velocity is set at $2-7 \mathrm{~cm} / \mathrm{s}$ per second $(\mathrm{cm} / \mathrm{s})$. Color Doppler flow is determined to be present when signals are reproducible.

Bowel loops are interrogated for presence or absence of mural blood flow and compared to adjacent loops within a region of interest (ROI). Performing US in stable and normal infants is very important. One should become familiar with normal neonatal bowel appearances to recognize the patterns of normal mural blood flow. The hyperemic patterns (Zebra pattern, "Y" appearance and circular flow) seen in infants with NEC and viable bowel are quite obvious and easy to recognize [3].

When bowel loops without mural blood flow are identified, the settings should be changed to more sensitive settings in an attempt to detect the slowest possible velocities. Using a large ROI to compare perfusion of adjacent bowel loops with color Doppler is very helpful. In case of absence of bowel wall perfusion, use of a smaller ROI is recommended.

Sample static images are obtained in each quadrant where the most loops are concentrated. Cine-loops or short videos should also be obtained. The exam is tailored to assess bowel with color Doppler US; however, all data are relevant. Data for each quadrant should be described separately and making notes during the examination is helpful. In case color Doppler US evaluation is not possible for a specific quadrant because of an increase in bowel gas, this should be mentioned in the report. After finishing the exam, I usually spend a few more minutes in the right lower quadrant. Statistically, it is the most affected quadrant. The examinations are then reported, as soon as possible, correlated with abdominal radiography and discussed with the neonatologist and pediatric surgeon.

The current literature has shown that absence of bowel perfusion is a sign of clinical deterioration and complication [3, 11-13]. However, over the years I have noticed that a relative decrease in mural flow does not necessarily indicate a poor prognosis. Some of these infants improve clinically, also showing improvement in bowel perfusion with shortterm US follow-up. Power Doppler or other more advanced Doppler techniques might also be helpful in this scenario. Research with contrast-enhanced US might also pave the way for its use in certain subsets of infants with NEC.

When neonates have more advanced disease and are hemodynamically stable, color Doppler US becomes easier to perform because there is decreased or absent peristalsis, and more fluid-filled bowel loops are noted in the most severely affected infants. On the other hand, the combination of increase in bowel gas and excessive peristalsis limits color Doppler US evaluation of intestinal blood flow because of artifacts. However, if peristalsis is present one can assume that bowel is viable.

It is well known that unstable patients should not undergo US evaluation. However, if a baby is stable and desaturates occasionally during the exam, one should stop and wait until oxygen saturation returns to normal in order to continue the examination.

It is very important to note that neonates on high-frequency ventilation are difficult to evaluate with color Doppler US. Also, a decrease in cardiac output and use of drugs such as vasopressors can cause difficulty in detecting color Doppler signals in these infants. Therefore, optimizing the settings is paramount. I recommend discussing these cases with the neonatologists to better understand the clinical and hemodynamic status of these infants. Follow-up exams within 12-24 h are helpful, in case there are equivocal findings in the bowel perfusion and abdominal radiography shows no evidence of pneumoperitoneum.

One should keep in mind that a large number of radiologists, technologists and trainees performing US in infants with NEC at the same institution could lead to a low sensitivity and specificity of this test. A lack of standard protocol also impacts in poor performance. Therefore, when implementing color Doppler US protocol, I would suggest limiting the number of radiologists and technologists involved and also promoting the use of standard reports.

I believe that getting enough exposure to the normal neonatal bowel appearances is paramount in the learning process. Subsequently, a small group at each institution can build the experience and understanding needed to assess these infants with NEC and, at the same time, work closely with neonatologists and surgeons.

We live in very exciting times with technology constantly evolving. There are new transducers available and we will soon be performing exams with $30-$ to $50-\mathrm{MHz}$ transducers. Abdominal US in NEC is a powerful bedside tool and we should continue to promote and incorporate it in patient care.

\section{Compliance with ethical standards}

Conflicts of interest None

\section{References}

1. Cuna AC, Reddy N, Robinson AL et al (2017) Bowel ultrasound for predicting surgical management of necrotizing enterocolitis: a systemic review and meta-analysis. Pediatr Radiol. https://doi.org/ 10.1007/s00247-017-4056-x

2. Lin PT, Stoll BJ (2006) Necrotising enterocolitis. Lancet 368:12711283

3. Faingold R, Daneman A, Tomlinson G et al (2005) Necrotizing enterocolitis: assessment of bowel viability with color Doppler US. Radiology 235:587-594

4. Epelman M, Daneman A, Navarro OM et al (2007) Necrotizing enterocolitis: review of the state-of-the-art imaging findings with pathologic correlation. Radiographics 7:285-305

5. Buonomo C (1999) The radiology of necrotizing enterocolitis. Radiol Clin North Am 37:1187-1198 
6. Miller SF, Seibert JJ, Kinder DL et al (1993) Use of ultrasound in detection of occult bowel perforation in neonates. J Ultrasound Med 12:531-535

7. Kodroff MB, Hartenberg MA, Goldschmidt RA (1984) Ultrasonographic diagnosis of gangrenous bowel in neonatal necrotizing enterocolitis. Pediatr Radiol 14:168-170

8. Vernacchia FS, Jeffrey RB, Laing FC et al (1985) Sonographic recognition of pneumatosis intestinalis. AJR Am J Roentgenol 145:51-52

9. Deeg KH, Rupprecht T, Schmid E (1993) Doppler sonographic detection of increased flow velocities in the celiac trunk and superior mesenteric artery in infants with necrotizing enterocolitis. Pediatr Radiol 23:578-582
10. Kim WY, Kim WS, Kim IO et al (2005) Sonographic evaluation of neonates with early-stage necrotizing enterocolitis. Pediatr Radiol 35:1056-1061

11. Silva CT, Daneman A, Navarro OM et al (2007) Correlation of sonographic findings and outcome in necrotizing enterocolitis. Pediatr Radiol 37:274-282

12. Muchantef K, Epelman M, Darge K et al (2013) Sonographic and radiographic imaging features of the neonate with necrotizing enterocolitis: correlating findings with outcomes. Pediatr Radiol 43: $1444-1452$

13. Aliev MM, Dekhqonboev AA, Yuldashev RZ (2017) Advantages of abdominal ultrasound in the management of infants with necrotizing enterocolitis. Pediatr Surg Int 33:213-216 